Article

\title{
Training for the Algorithmic Machine
}

\author{
Stefka Hristova \\ Department of Humanities, Michigan Technological University, Houghton, MI 49931, USA; E-Mail: shristov@mtu.edu
}

Submitted: 23 October 2021 | Accepted: 25 November 2021 | Published: 6 April 2021

\begin{abstract}
In thinking about the ubiquity of algorithmic surveillance and the ways our presence in front of a camera has become engaged with the algorithmic logics of testing and replicating, this project summons Walter Benjamin's seminal piece The Work of Art in the Age of Its Technological Reproducibility with its three versions, which was published in the United States under the editorial direction of Theodore Adorno. More specifically, it highlights two of the many ways in which the first and second versions of Benjamin's influential essay on technology and culture resonate with questions of photography and art in the context of facial recognition technologies and algorithmic culture more broadly. First, Benjamin provides a critical lens for understanding the role of uniqueness and replication in a technocratic system. Second, he proposes an analytical framework for thinking about our response to visual surveillance through notions of training and performing a constructed identity-hence, being intentional about the ways we visually present ourselves. These two conceptual frameworks help to articulate our unease with a technology that trains itself using our everyday digital images in order to create unique identities that further aggregate into elaborate typologies and to think through a number of artistic responses that have challenged the ubiquity of algorithmic surveillance. Taking on Benjamin's conceptual apparatus and his call for understanding the politics of art, I focus on two projects that powerfully critique algorithmic surveillance. Leo Selvaggio's URME (you are me) Personal Surveillance Identity Prosthetic offers a critical lens through the adoption of algorithmically defined three-dimensional printed faces as performative prosthetics designed to be read and assessed by an algorithm. Kate Crawford and Trevor Paglen's project Training Humans is the first major exhibition to display a collection of photographs used to train an algorithm as well as the classificatory labels applied to them both by artificial intelligence and by the freelance employees hired to sort through these images.
\end{abstract}

\section{Keywords}

algorithmic culture; artificial intelligence; critical theory; facial recognition; selfies; surveillance; technological reproducibility

\section{Issue}

This article is part of the issue "Critical Theory in a Digital Media Age: Ways Forward" edited by Robert E. Gutsche, Jr. (Lancaster University, UK).

(C) 2021 by the author; licensee Cogitatio (Lisbon, Portugal). This article is licensed under a Creative Commons Attribution 4.0 International License (CC BY).

\section{Introduction}

Today one's face has come to replace one's fingerprint as the primary unit of identification. Currently, there are over 30 companies across different sectors such as banking, beauty brands, food and beverage brands, and hotels that are developing and testing facial recognition technologies ("Facial recognition," 2019). Among them is the retail giant Amazon which in 2018 unveiled its affordable software for facial recognition Rekognition. According to Amazon's website:
Rekognition is an image recognition service that detects objects, scenes, and faces; extracts text; recognizes celebrities; and identifies inappropriate content in images. It also allows you to search and compare faces. Rekognition Image is based on the same proven, highly scalable, deep learning technology developed by Amazon's computer vision scientists to analyze billions of images daily for Prime Photos. (Amazon, 2020a)

For under $\$ 10$, now anyone can deploy this computervision, deep learning Al driven tool to identify 'targets' 
and 'innocents' based on photographs or video footage (Amazon, 2020a). Rekognition has been deployed in a variety of contexts. For example, the Oregon Police Department uses the software to identify 'persons of interest' (Fazzini, 2018); Aella Credit on the other hand has deployed the software as means of identification of potential borrowers in emerging markets while Daniel Wellington relies on this technology to identify customers who come to return items bought in their highend jewelry stores (Amazon, 2020b). The customer list posted on Amazon's website also boasts working with the dating company Soul to "to detect objectionable content before it's posted while minimizing the need of human involvement" and with the children-oriented app Go Girls, the photo service Sen Corporation, the summer camp platform CampSite. My point is that the use of facial recognition software, be it Amazon's or that developed by one of the other tech giants such as Google and Facebook, has become a ubiquitous part of our everyday life. It is used in digital and analog spaces to identify and track all of us adults as well as our children.

The Rekognition software enables the recognition of both loyal customers and those deemed undesirable. Rendered through the Rekognition algorithm, the individual becomes either a celebrity or a stalker; in other words, either a legitimized public figure or a criminalized private citizen. Rekognition is indeed being sold to celebrities as a way to manage fans and stalkers and this dichotomy is anchored in the public description of the algorithm itself. In a sense then, algorithmic surveillance is constantly categorizing the humans that it detects into honorific and repressive categories. The repressive use of the algorithm is particularly problematic because of the perceived veracity and actual factual inaccuracy with which it operates. Recently, Pasco County Sherriff's office deployed a biased algorithmic predictive system that "generates lists of people it considers likely to break the law, based on arrest histories, unspecified intelligence and arbitrary decisions by police analysts" and then sends deputies to interrogate the targeted individuals (McGregory \& Bedy, 2020). In verifying the criminal status of individuals, facial recognition has also often proven to be inaccurate; this inadequacy has been demonstrated by multiple studies and incidents. Notably, Robert Julian-Borchak Williams was arrested based on a comparison of two photographs taken by a surveillance camera and his driver's license (Allyn, 2020). The match was justified by an argument that algorithms are objective and can identify criminals better than humans based on an assessment of similarity between visual images. This was one of few cases in which the "police admitted that facial recognition technology, conducted by Michigan State Police in a crime lab...prompted the arrest" (Allyn, 2020). Algorithms are deployed in all aspects of our lives and have come to guide biopolitical decisions on our behalf. What is different here is the biopolitics of everyday life are now entrusted in a technological system that is further curtailing the role of humans as the decision makers.
Human agency, in other words, is relegated to the production of 'raw' material that is to be gathered, accessed, categorized, and acted upon through algorithmic means on behalf of technocratic corporations.

As their training base, facial recognition algorithms often use 'scraped' consumer photographs (i.e., taken from the Internet without notifying users) such as selfies and digital images as well as state-issued photographs such as those used on driver licenses. Consumer photographs posted on Amazon Prime Photos were used without the explicit permission of the users who took and uploaded them for the training of the Rekognition algorithm. The Digital photographs became the basis for algorithmic surveillance and as such, they permeated not only the social media landscape but also the space of algorithmic culture more broadly. As the windows to our souls are reshaped into iris scans and the pictures of our minds become faceprints, it is important to note not only when and how these scans and prints are used to assess the risk that one poses to society but also when and how our eyes and faces became measurable windows/pictures in the first place.

Algorithmic culture functions as a technological culture rather than simply as a digital media culture. Digital culture has traditionally articulated to the ways in which digital media has shaped culture, whereas in the context of algorithmic culture, digital and algorithmic technologies well beyond media are shaping society. As I have argued, "[I]n the context of an algorithmic culture, then, it is increasingly important to understand the ways in which algorithmic structures through recognition, calculation, automation, and prediction are shaping everyday life" (Hristova, 2021, p. 3). The term technological culture, as coined by Jennifer Slack and Gregory Wise (2015, p. 9), broadly describes the ways:

Culture has always been technological, and technologies have always been cultural. Technologies are integral to culture and not separate from it.... Human culture has always existed in relation to what we understand to be technologies: from voice, stone, and fire, to clock, computer, and nanotechnology.

As such, the contemporary moment can be seen as the orientation of a culture towards a new technologynamely that of algorithmic technologies and should be discussed in the context of technological culture alongside notions of mediated culture. The term algorithmic culture "draws attention to the reality that culture is increasingly explained by, responsive to, and shaped in and by the pervasive work of algorithms" (Slack \& Hristova, 2020, p. 18). Algorithmic culture thus accounts for the ways in which this new form of digital technology is changing all aspects of everyday life, not just our engagement with media. The study of algorithmic culture then, as articulated through its technological and cultural aspects, necessitates critical perspectives that grapple with the nexus of new technological developments, 
politics, economics, and practices of resistance. Arguably, the current moment is not the first time that we have encountered the problem of pervasive surveillance coupled with the proliferation of right-wing regimes worldwide. Indeed, critical theory as articulated by Theodore Adorno and Walter Benjamin emerged under a similar historical context and is indeed quite relevant for addressing our contemporary predicaments. Benjamin's work offers important concepts that "differ from others in they are completely useless for the purposes of fascism. On the other hand, they are useful for the formulation of revolutionary demands in the politics of art" (Benjamin \& Jennings, 2010). Moreover, Benjamin's ruminations on technology offer myriad concepts that help us untangle the technological transformations in the context of the increased presence of right-wing ideology and right-wing authoritarian governments. For Benjamin, understanding the ways in which visual apparatuses construe us and actively training to perform a desired identity in the context of technological surveillance holds the possibility of technological disruption. In other words, being knowledgeable of how technology frames us allows for a more intentional presentation of the self, which in turn holds the potential to render technologies themselves impotent or useless to autocratic regimes of power.

In the context of algorithmic culture, surveillance has become an increasingly important topic (Benjamin, 2019; Gates, 2011; Monahan, 2006; Noble, 2018; Pasquale, 2015). In exploring the ways in which our presence in front of a camera has become engaged with the algorithmic logics of testing and replicating, I summon Walter Benjamin's seminal piece The Work of Art in the Age of Its Technological Reproducibility with its three versions, which was published in the United States under the editorial direction of Theodore Adorno (Benjamin, 2002, 2003; Benjamin \& Jennings, 2010). The first version was written in 1935, while the second version of the essay from 1936 is a "revision and expansion...of the first version...[and] represents the form in which Benjamin originally wished to see the work published" (Benjamin, 2002, p. 122). The third and most popular in the United States version, which Benjamin completed in 1939, was modified based on the editorial input of Adorno who facilitated the translation, publication, and popularization of this work (Benjamin, 2003, p. 270). The third version backtracks some of the celebratory stances awarded to the notion of replication and reproducibility (that are found in the first two versions) and bears clear traces of Adorno's fascination with the authentic as well as his disdain for mass art. It also moves away from an understanding of visual and visualization technologies and towards a narrower articulation of visual media. In this essay, I highlight two of the many ways in which the first and second versions of Benjamin's influential essay on technology and culture resonate with questions of photography and art in the context of facial recognition technologies and algorithmic culture more broadly. First, Benjamin provides a critical lens for understanding the role of unique- ness and replication in a technocratic system. Second, he proposes an analytical framework for thinking about our response to visual surveillance through notions of training and of performing a constructed identity-hence, being intentional about the ways in which we visually present ourselves. These two conceptual frameworks help to articulate our unease with a technology that trains itself using our everyday digital images in order to create unique database identities that further aggregate into elaborate typologies and to think through a number of artistic responses that have challenged the ubiquity of algorithmic surveillance. Adapting Benjamin's conceptual apparatus and his call for understanding the politics of art, I focus on two projects that powerfully critique algorithmic surveillance. Leo Selvaggio's URME (you are me) Personal Surveillance Identity Prosthetic offers a critical lens through the adoption of algorithmically defined three-dimensional printed faces as performative prosthetics designed to be read and assessed by an algorithm. Kate Crawford and Trevor Paglen's project Training Humans is the first major exhibition to display a collection of photographs used to train an algorithm as well as the classificatory labels applied to them both by $\mathrm{Al}$ and by the freelance employees hired to sort through these images.

\section{Replication for Whom: Humanistic and Technological Assemblages}

Benjamin articulated his well-known concept of reproducibility as operating on two different levels: One in which "objects made by humans could always be copied by humans and another in which the reproduction was articulated through technology and thus became 'technological reproduction"' (Benjamin \& Jennings, 2010, p. 12). This second mode of reproducibility was articulated through the emergence of the woodcut, became amplified through the technology of lithography, and culminated in the introduction of photography, which was seen as a technology further displacing the human from the process of reproduction by delegating the process of "pictorial reproduction...to the eye alone" (Benjamin \& Jennings, 2010, p. 14). In this context, the eye follows the primary mediation of the original conducted by the lens. In terms of algorithmic culture, the processes of reproduction and detachment are further amplified and dehumanized. Indeed, this dehumanization emerges as a fundamental process that accompanies the move away from the prehistoric connection between technology and ritual towards a machine age driven by technological reproducibility. Writing prophetically in the 1930 s, Benjamin foresees the continued displacement of the human and humanity towards technological autonomy. In thinking about the distinction between ritual-based and machine-based technologies, he wrote:

Whereas the former made the maximum possible use of human beings, the latter reduces their use 
to the minimum. The achievement of the first technology [seen in a prehistoric context for example] might be said to culminate in human sacrifice; those of the second [rooted in the Machine age], in the remote-controlled aircraft which needs no human crew. The results of the first technology are valid once and for all....The results of the second are wholly provisional (it operates by means of experiments and endlessly varied test procedures). (Benjamin, 2002, p. 107)

Under the auspice of photography, the process of reproduction became one that is exclusively visual and continuously technological. The eye here is further displaced as photography was seen as "bringing out aspects of the original that are accessible only to the lens" (Benjamin \& Jennings, 2010, p. 14). Like the remote-controlled aircraft without a human pilot, replication through photographic means is now directed not for the human eye itself but rather it is distilled into a set of features that are accessible only to the lens and the algorithm: faces become faceprints, eyes become iris scans. Trevor Paglen has theorized the emergence of images in relation to machine learning and $\mathrm{Al}$ as "invisible images" embedded in "machine-to machine seeing" in which "digital images are machine-readable and do not require a human in the analytic loop" (Paglen, 2019, p. 24). Whereas for Benjamin visual film-based technology (photography and film) revealed optical unconscious properties unattainable to "natural optics" such as "enlargement or slow motion" but are ultimately made perceptible to human vision (Benjamin, 2002, p. 102). In the contemporary context, however, visual algorithmic technology reveals properties that are even less unattainable by human perception as they articulate a set of data points meaningful only to algorithms. For example, an iris scan consists of at least 240 data points and thus distills the world in a manner that is understood by machine vision and machine knowing (learning). While the photograph captured faces, an algorithmdriven camera now sees face models that are meaningless to human vision.

This translation of face-to-face model in the context of facial recognition algorithms is evident in reading Amazon Rekognition's developer guide where a face model becomes defined as a bounding box and further given coordinates for the expected elements: eyes, nose, mouth:

\footnotetext{
"FaceModelVersion": “3.0,”

"SearchedFaceBoundingBox": \{

"Height": 0.06333333253860474,

"Left": 0.17185185849666595 ,

"Top": 0.7366666793823242 ,

"Width": 0.11061728745698929

\} ,

"SearchedFaceConfidence": 99.99999237060547
}

Amazon's surveillance software articulates personhood first as the presence of a face and second through the existence of posture. Furthermore, a face is conceived as consisting of a left eye, a right eye, a nose, the right side of one's mouth, and the left side of one's mouth. The face thus becomes the locus of personhood in the context of algorithmic surveillance (Amazon, n.d.). Through this process of technologically reproducing people through visual capture of either subjects or photographs of subjects, the image is distilled into image-data. This distillation obfuscates the relevance of the real, the original beyond its datafied existence. Within the context of facial recognition technology, this process informs the technological articulation of both the input and output of the technological reproduction process.

Portraits, selfies, and photographs of people, in general, are particularly susceptible to this transformation as bodies in front of a camera are captured by its lens and further translated into data for an algorithm. The endpoint of the camera is no longer a photograph. It is data. The lens then produces not an image but a dataset. Facial recognition algorithms use consumer photographs such as selfies and digital images as well as state-issued photographs such as those used on driver licenses as their training base. Once within the sphere of the algorithm, the human body is relevant only as data and the image itself becomes a useless intermediary. These data-points are articulated in big data structures from which typologies emerge. Thus, the individual who stood in front of the camera for a portrait or selfie, or simply walked in front of a consumer or commercial camera is simply understood in algorithmic terms, as an example of a larger 'measurable type' (Cheney-Lippold, 2017).

In an algorithmic culture, the authentic individual is replaced with an entity enthralled in a projected typology in which common habitual traits are replicated and reproduced. In other words, the uniqueness of individuals or their aura is the main fuel of the algorithmic machine. The machine relies on difference and differentiation in order to trace unique database ids through time and space. Benjamin's critique on the insistence of holding on to the notion of authenticity, of customization, of uniqueness is quite powerful. In an algorithmic culture, if the original is already a replica without an aura, then the process of technological reproduction is disempowered. For the algorithm to work, individual behavior must demonstrate patterns or 'trends' but it also much be distinct enough as to articulate a separate data point or big data. In other words, individuation is useful to an algorithm as it provides a point into a set of big data. Without multiple individual points, there is no big data, and thus the algorithm has nothing to work with. The individuation we are currently afforded is a superficial one-one that is based on quantitative difference: We can buy a blue case for our similar iPhone, or choose to purchase a pink Rumba to clean our floors. We, however, are seen as static unique entries that wear pink or blue (variation) but remain constant and unique at the same time. Benjamin 
proposes an alternative framework in which individuals, not just art, might consider operating as consciously reproducible entities without an aura. In a post-aura technological landscape, accepting a level of sameness on a mass scale can defeat the big data impetus of algorithms and thus render us useless to this technology. The level of sameness here addresses the attempt of algorithms to reconstruct us as digital selves, as unique digital identities within group clusters. In a culture of corporate standardization and surveillance capitalism, algorithms attempt to reinstate algorithmic aura by defining the terms that make us unique in a way that is inaccessible to us (Zuboff, 2019). What is authentic and what is replicable about our own selves and our behavior is no longer a choice that we as humans can make but is rather relegated to an algorithmic calculation. Our algorithmic aura is neither comprehensible nor accessible to ourselves.

This theme of the non-original is visualized in Leo Selvaggio's project URME (you are me) Personal Surveillance Identity Prosthetic in which he offered his own face as a 3D printed mask in order to flood the streets with Leos as far as facial recognition technologies are concerned. Selvaggio's project mobilizes reproducibility, reproduction, and replication as a political tactic against the reappearance of the algorithmic aura and its dominating uniqueness. With the prosthetic, while the human eye is able to detect the mask, the replication for the algorithmic eye is flawless and the algorithm 'sees' a series of Leos. This distinction is important. Masks traditionally have been seen as technologies of resistance. As Zach Blas (2013) wrote, "The mask is the most popular implementation of defacement, a celebration of refusal and transformation." Masks are valuable defacement mechanisms in a human and algorithmic context. Selvaggio's project both builds upon and moves away from masks as a mechanism for defacement and towards an exploration of masks as standardized humanoid surfaces. His work is a prime example of an artistic anti-surveillance camouflage practice that asks individuals to explore the practice of algorithmic reproducibility as an act of resistance. This project "involves the design of masks that are eerie reproductions of his own face, potentially spawning swarms of expressionless doppelgangers moving through the street" (Monahan, 2015, p. 166). These masks were tested with Facebook's recognition systems and proven to trigger the detection of Selvaggio's face. Selvaggio's narration of the project is quite poignant:

I imagine a future where everyone wears my face, literally. Take a moment to consider this future. As you walk down the street to the subway, you pass by me over and over and over again. The sliding doors of the train open to a swarm of Leos. (Selvaggio, 2015, p. 165)

Thus, forgoing the process of individuation renders the face when understood as face model useless.

\section{Training for the Camera: Constructing Identities in the Age of Machine Vision}

Algorithms are trained on our images. This primary framework for training is precisely what Amazon's Recokgnition software deployed without the knowledge of the Internet users whose faces were used for the establishment of surveillance categorizations. If we are to understand ourselves as constantly being subjected to processes of surveillance and further replication through the lens of algorithmic calculations, we must consider the intentionality underlying the adapting of our everyday behavior. We should consider training ourselves in order to understand how the algorithms work in order to resist this new apparatus of surveillance. In an age where technology is further displacing the idea of humanity away from authenticity and towards replicability with the illusion of an algorithmic aura, Benjamin sees film as a training ground for resistance through the medium's ability to help us to understand the mechanism that guides reproduction and learn how to be present for the technological apparatus. With regard to the potentiality of film, Benjamin wrote that "the function of film is to train human beings in the apperceptions and reactions needed to deal with the vast apparatus whose role in their lives is expanding almost daily" (Benjamin, 2002, p. 108). His distinction between the stage actor and the film actor is helpful here for understanding the new way in which our replicas percolate in the algorithmic technological landscape. For the film actor, the "original form, which is the basis of the reproduction, is not carried out in front of a randomly composed audience but before a group of specialists" (Benjamin \& Jennings, 2010, p. 22). This process enables training with experts of the technology and thus responding to the primary modality of algorithms training on humans without the permission or even knowledge of the ladder. As photography, film, and social media bleed into algorithmic facial recognition systems, a similar call is being issued by prominent artists today. For example, Paglen powerfully noted that:

The point here is that if we want to understand the invisible world of machine-to-machine visual culture, we need to unlearn how to see like humans. We need to learn how to see a parallel universe composed of activations, keypoints, eigenfaces, feature transforms, classifiers and training sets. (Paglen, 2019, p. 27)

Understanding machine vision is crucial in order to be able to train and perform identities suited to this new technological landscape. Much like the actor, each of one of us is encouraged to understand and intentionally train in front of the algorithmic apparatus. In the context of film, or rather filming, the actor practices the act until it is made perfect for the lens: a "single cry for help, for example, can be recorded in several different takes" (Benjamin \& Jennings, 2010, p. 22). Thus, for the 
film actor, being in front of the camera for the film actor is a "performance produced in a mechanized test" of a premediated fictional role and an intentionally constructed identity. Intentionality here is key because the film actor is allowed to train with the help of experts whereas workers are subjected to the same exact tests and judgment but participate in them 'unaware.' Furthermore, Benjamin warned that "the film actor performs not in front of an audience but in front of an apparatus [in which] the film director occupies directly the same position as the examiner in an aptitude test" (Benjamin \& Jennings, 2010, p. 22). It is this awareness and intentionality that bring humanity back into the process as "for the majority of city dwellers, throughout the workday in offices and factories, have to relinquish their humanity in the face of the apparatus" (Benjamin \& Jennings, 2010, p. 23). In a film test, it is only the performance of the character that is captured in this test, not the authenticity of the actor.

Selvaggio's project engages precisely with this intentional performative model. He suggests that "when we are watched we are fundamentally changed. We perform rather than be" (Katz, 2014). Thus, this performance thus is not an act of hiding, it is an act of modifying one's performance for the camera, much like an actor performing a character would. Selvaggio revealed the strategy behind his project as one that "rather than hide a face, substitute[s] it" (Selvaggio, 2015, p. 174). This substitution is articulated in the context of facial recognition technologies deployed precisely in relation to crime.

For Benjamin, the film apparatus provided a training ground for the ways in which one's mirror image became replicated and distributed across networks. His observations could be translated to the context of digital photography and algorithmic surveillance, where the selfie has become the mode par excellence of self-broadcast to the world via social media networks and algorithmic surveillance is seen as the most pervasive modality of non-consensual capture and datafication of selfies, digital portraits, and street photography. The notion of being aware of the ways in which the camera and the algorithm translate our physical selves into reproducible dataselves is crucial here. Being unaware of the surveillance regimes in which we are embedded removes individual agency. Thus, it is critical to understand how algorithmic surveillance works and how one can test in front of it and perfect a performance of an identity that is intentionally crafted to respond to the technological apparatus. Unfortunately, we are asked to consider both conscious and unconscious behavior at a micro-level. Consider the millisecond you spend while scrolling on Facebook while looking at sponsored content or the ways in which you raise your eyebrows while reacting to digital content. One implies an interest in a product and sells your potential consumer power. The other outright renders the consumer into a product to be evaluated: If your eyes are too close to your eyebrows your Body Mass Index becomes elevated and your health score decreases. The more we know about the metrics that are judging us the more we can intentionally counter them.

The ubiquity of surveillance coupled with its invisibility or rather seamless blending with reality deeply resonates with Benjamin's observation that "the apparatusfree aspect of reality has....become artifice, and the vision of unmediated reality the Blue Flower in the land of technology" (Benjamin \& Jennings, 2010, p. 28). A vision of unmediated reality is thus seen as an inaccessible, romanticized ideal as the Blue Flower represents "the unattainable object of the romantic quest, the incarnation of desire" (Hansen, 1987, p. 204). This does not mean that one must surrender to the idea of technological mediation and should abolish efforts to challenge the technological and political systems that drive mediation. Rather, Benjamin suggested, an open acknowledgment of our predicament, an awareness of the way that it 'sees' us, and an effort to mindfully attempt to craft our presence.

Mitra Azar's (2018) work on algorithmic facial images is of particular relevance here. Azar has made a compelling argument that "when a selfie becomes mediated by new tracking technologies for security system and entrainment based on face-recognition algorithms, the selfie becomes an 'Algorithmic Facial Image"' (Azar, 2018, p. 27). In the appropriation of the photograph from selfie to a facial image, Azar noticed an important change:

If in the early 2000s the selfie seemed to be characterized by a certain degree of (calculated) spontaneity, an analogically constructed liveness and a form of human agency, this new form of selfie is rather defined by trackability, its algorithmically constructed liveness, and its non-human agency. (Azar, 2018, pp. 27-28)

In this transition, the camera itself becomes in the words of Deleuze and Guattari a 'faciality machine' (Deleuze \& Guattari, 1987 p. 199). The algorithmic machine that I am referring to in this project is indeed a faciality machine. What is notable here is the emergence of the selfie as a particular type of performance for the camera facing us rather than the world and the potentiality for the augmentation of this act when the visualization technology becomes understood as a faciality machine. In other words, we have already trained to perform a 'selfie' for the camera and are now in the moment of retraining once more-this time, in the context of algorithmic visuality.

Kate Crawford and Trevor Paglen's project Training Humans highlights precisely the ways in which selfies, portraits, and state-issued identification have been harnessed in the training of facial recognition algorithms without the knowledge of the people in these photographs. Training Humans was "the first major photography exhibition devoted to training images: the collections of photos used by scientists to train Al systems how to 'see' and categorize the world" (Crawford \& 
Paglen, 2020). As the authors note, they are reintroducing into the gallery photographs that "aren't really meant for humans [as] they're in collections designed for machines" (Crawford \& Paglen, 2019). Here Crawford and Paglen exposed the inner workings of algorithmic classification and, in a sense, acted as the experts who allowed audiences to understand and train for the new algorithmic machine. The exhibit provided historical context about the ways in which anthropometrics and biometrics have historically been deployed in the articulation of human typologies. They further displayed the images used to create algorithmic classifications uncovering the duality of the photograph as an honorific and repressive entity. The most powerful part of this project is the real-time visualization of the algorithmic decisionmaking process as it evaluates the gender, age, and emotion of the people it 'sees' (Crawford \& Paglen, 2019). According to Crawford, they:

Wanted to engage directly the images that train Al systems, and to take those images seriously as a part of a rapidly evolving culture. They represent the new vernacular photography that drives machine vision. To see how this works, [they] analyzed hundreds of training sets to understand how these 'engines of seeing' operate. (Crawford \& Paglen, 2020)

Furthermore, Crawford characterized this training process as two-pronged: as everyday photographs training algorithms and as algorithms training humans how to behave. Training Humans, alongside Crawford and Paglen's Excavating Al project, raises an important question about the lack of awareness by the people in the photographs about the ways in which their faces are harnessed for algorithmic testing. Unlike Benjamin's actor and much like his worker, those posing for a selfie or a digital image were often unaware of the algorithmic classificatory systems they helped shape and ultimately became trapped by: "Harvesting images en masse from image search engines like Google, ImageNet's creators appropriated people's selfies and vacation photos without their knowledge, and then labeled and repackaged them as the underlying data for much of an entire field" (Crawford \& Paglen, n.d.). What Crawford and Paglen's projects reveal is not only how the actor or worker is trained but also how the machine apparatus, the technology is 'learning' as well. In other words, the training goes both ways. The training of Al requires "vast amounts of data contained within datasets made up of many discrete images" (Crawford \& Paglen, n.d.). The training of the human in front of the lens requires knowledge and intentionality.

Alan Sekula has eloquently argued that photographs have always participated in an honorific and repressive systems of representation as the portrait and the mugshot have been intimately connected since the invention of photography (Sekula, 1986, p. 10). This connection that as Sekula argues introduced "the panoptic principle into daily life" (Sekula, 1986, p. 10) has been further amplified in the context of Al where the number of images analyzed is well into the several hundred million as seen by 2009's ImageNet project (Deng et al., 2009). While the scope of "scraped" images is impressive, so is the extensive classificatory schema behind it. This classificatory schema, developed through 'crowdsourcing' on Amazon's labor marketplace Mechanical Turk, is then reflected back to the unsuspected users of the digital world. As Crawford and Paglen note, here not only race, gender, and economic status are encoded to algorithmic data and back as cultural identity, but so are value judgments about people:

As we go further into the depths of ImageNet's Person categories, the classifications of humans within it take a sharp and dark turn. There are categories for Bad Person, Call Girl, Drug Addict, Closet Queen, Convict, Crazy, Failure....There are many racist slurs and misogynistic terms. (Crawford \& Paglen, n.d.)

The classification schema was developed to aid the recognition and sorting processes driven algorithms and benefits the owners of the technological apparatuses and not the humans who were 'processed' as training data. In Training Humans, they further provide an extensive genealogy specific to the ways in which algorithmic facial recognition participates in narratives of human classification. As such this project is a direct extension of what Sekula, as well as Crawford and Paglen, trace to be a genealogy of eugenics rooted in the 19th century phrenology and physiognomy work of Francis Galton, Alphonse Bertillon, and Cesare Lombroso (Crawford \& Paglen, 2019, p. 21). The distillation of images into data for the purposes of algorithmic capitalist surveillance is yet the latest instance of the enmeshment of photography with eugenics. Crawford and Paglen's project exemplifies par excellence the claim that Lea Laura Michelsen has aptly made, "Digital biometrics can be perceived as a physiognomic renaissance" (Michelsen, 2018, p. 37).

Art projects that enable the public to see how they are being judged by algorithms have been developed not only for art galleries but also through digital platforms with greater access. One example is Tijmen Schep's How Normal Am I interactive documentary project (Schep, 2020). In it, the audience is asked to turn on their camera and is guided through a series of algorithmic decisions while Schep narrates the inner workings of facial recognition. He reveals the ways in which beauty is judged on platforms such as Tinder, where people with similar scores are considered to be a match, and unpacks how health insurance industries use facial recognition to predict BMI indexes and thus assess health risk. In this project, the audience is also given the opportunity to train for the algorithm: "By giving access to your webcam you can also experience how these Al systems rate your own face" (Schep, 2020). The experience is coupled with useful tips; for example, raising one's eyebrow leads the 
algorithms to assume a greater BMI index and thus the risk of obesity. Both Training Humans and How Normal am I allow for subjects in front of the camera to test their behavior and see the different outcomes live. They are given tips on how to perform and then are allowed to see if their behavior is gaged based on their expectations. The training in front of the camera is responsive and guided by experts who understand the inner workings of the algorithm. The training here begins in the context of art and raises awareness about the ways in which assessments are made about our conscious and unconscious behavior.

Understanding and regulating the processes of data gathering processes as well as algorithmic development practices are crucial components for the development of a more equitable algorithmic culture: A culture that asks how apparatuses of assessment are created and indeed can them move forward to challenge them and perhaps call for their abolishment. While structural resistance is absolutely vital, so is micro-level training on how we are being judged by facial recognition platforms. Politicized algorithmic art allows us to bring back intentionality and awareness in front of the camera and to practice the ways in which to carry and present ourselves in front of this new capitalist surveillance assemblage in a safe space. In other words, by engaging with projects that are critical of facial recognition, we can start to understand and adapt to the inner workings of this new modality of technological reproduction and also challenge the deployment of these technologies altogether. If we are going to live in an increasingly algorithmic world, we must adapt to it mindfully in the meantime and resist the entrenchment of technocratic political orders in the long term.

\section{Disruptive Practices: Unleashing the Revolutionary Potentials of Art and Performativity}

The rise of $\mathrm{Al}$ and facial recognition surveillance has yet again elicited questions about the ways in which the algorithms can be designed to be more accurate, less biased, subjected to legal systems, decoupled from authoritarian regimes, and last but not least individually resisted. For Benjamin, technological reproduction offered an escape from "enslavement" and this liberation was to come "only when humanity's whole constitution has adapted itself to the new productive forces which the second technology has set free" (Benjamin, 2002, p. 108). This freedom to play once "liberated from drudgery" was seen as possible only "when the collective makes this technology it's own" (Benjamin, 2002, p. 124). The discourse on technological liberation from chores for the sake of convenience and play still resonates today in discourses about how now computing and autonomous technology are allowing more playtime. This celebratory stance towards collective ownership of technology that takes at its heart the rejection of authenticity and ritual and the embrace of popular and replicable is challenged by Theodor Adorno (n.d.) in a letter to Benjamin. Adorno rightfully insisted on considering the larger economic structure that makes mass art possible. In the contemporary context, visual technologies ranging from digital photography to algorithmic surveillance are not democratized but rather lay in the hands of few corporations. The reproducibility that they offer under the guise of play is articulated in terms that are useful for the machines themselves and the capitalist frameworks of alienation in which they operate. On the other hand, Benjamin sees the politics of art or further the politicizing of art as a powerful antidote to authoritarian and exploitative regimes (Benjamin \& Jennings, 2010, pp. 12, 36).

The idea that art can be a powerful agent of change has been challenged by critics of state and capitalism surveillance. As Torin Monahan has aptly noted, in the age of increased surveillance, there has been a rise in antisurveillance camouflage in the form of artistic projects and products centering on "masking of identity to undermine technological efforts to fix someone as unique entity apart from the crowd" (Monahan, 2015, p. 159). He has questioned the effectiveness of such projects:

Anti-surveillance camouflage of this sort flaunts the system, ostensibly allowing wearers to hide in plain sight-neither acquiescing to surveillance mandates nor becoming reclusive under their withering gaze. This is an aestheticization of resistance, a performance that generates media attention and scholarly interest without necessarily challenging the violent and discriminatory logics of surveillance societies. (Monahan, 2015, p. 160)

Monahan proceeded to situate this right to hide in relation to the surveillance practice of the state which has embraced the right to look and denied the right to look back. This position on the uselessness of art has been countered by a strong justification of the role of surveillance art in the larger cultural landscape. Monahan insists on the importance of challenging the institutional, economic, and legal systems in which algorithmic surveillance operates, and rightly so. However, art offers yet another track of resistance that does not assume the erasure of other oppositional positions, but rather amplifies the struggle against these normative technological apparatuses. As Elise Morrison has written:

\begin{abstract}
Surveillance art, as a genre of political activism and performance, combats the common tendency within surveillance society to succumb to a kind of amnesia of convenience, an ambivalent state in which the majority of user-consumers are willing to forget or look past the risks of using surveillance technologies in prescribed ways because of perceived economic, political and social gains. (Morrison, 2015, p. 126)
\end{abstract}

The dialectic here is one that questions the role of the arts in conversations about technology and culture. I side 
here with Morrison's sentiment that art allows for a critical framework through which naturalized relations can be brought back to a reflective practice. I think that the greatest contribution to the artistic projects described above is their contemplative nature or to come back to Benjamin's work - their intentionality in situating ourselves in the position of the aware film actor rather than the unaware mechanized worker.

Whereas artistic practice is already embedded in critical reflective practice, everyday posturing in front of the digital mobile camera is hardly so. With regards to art, Torin Monahan asked a poignant question: "By claiming what can be framed as a 'right to hide,' instead of a 'right to look,' what, exactly, does anti-surveillance camouflage perform" (Monahan, 2015, p. 166). In thinking about mass strategies for addressing algorithmic surveillance, I want to address both the potential role of training to look back at the camera as well as training to hide from the camera. Reflective posturing could be seen as an example of resisting surveillance capitalism through the paradigm of the right to hide. The activists have also been deploying facial recognition as an apparatus reinstating the 'right to look.' Among them is Christopher Howell who has turned the camera back to the Portland police officers "since they are not identifying themselves to the public and are committing crimes" (Howell, Strong, Ryan-Mosley, Cillekens, \& Hao, 2020). Resistance to the technological panopticon created by facial recognition algorithms must be multi-fold and multi-directional. Through individual reflective practice based on awareness of the assessment mechanism behind the camera or through collective reflective action in turning the camera onto the surveyors themselves, intentional visuality might just be a power full for resisting the rise of both the surveillance state as well as surveillance capitalism.

\section{Conclusion}

As we move between a digital media world in which digital selves are articulated through algorithms for the purposes of advertising to an algorithmic culture where algorithms monitor and evaluate our conscious and unconscious behavior through thousands of cameras embedded in both public and private spaces, it is crucial to continue to explore modes of critique and resistance. Walter Benjamin's first and second versions of this famous essay The Work of Art in the Age of Its Technological Reproducibility offer an important apparatus for challenging algorithmic surveillance. Benjamin's assessment of the role of reproduction on one hand and training in front of the camera on the other offer important insights into our contemporary conditions. His writing on art and film in the context of fascism is indeed deeply relevant to an analysis of surveillance art in the context of a global proliferation of right-wing authoritarian regimes. Benjamin offers a powerful critique of the ways the camera reproduces not just art but also human behavior and one might say specters of the humans themselves and in this process excises the original-be it again the artwork or the human caught in the reproduction loop. One of the mechanisms for challenging this technocratic framework that he offers is the emphasis on reflection and intention. This reflection process entails an intentional reversal of the basic assumptions that structure algorithmic technology and thus the introduction of deflective methods of resistance. Some of these deflective methods have been harnessed by contemporary artists as a critique of algorithmic culture. Just as algorithms look for individual data points, artists challenge our algorithmic aura. Just as the algorithm trains on humans, artists help humans in training for the algorithm. Until we can dismantle the contemporary algorithmic panopticon, a game of hide and deflect might be in order.

\section{Conflict of Interests}

The author declares no conflict of interests.

\section{References}

Adorno, T. (n.d.). Letter to Benjamin. Art Theory. Retrieved from https://theoria.art-zoo.com/letterto-benjamin-theodor-adorno

Allyn, B. (2020, June 24). 'The computer got it wrong': How facial recognition led to false arrest of black man. NPR. Retrieved from https://www.npr.org/2020/06/ 24/882683463/the-computer-got-it-wrong-howfacial-recognition-led-to-a-false-arrest-in-michig

Amazon. (n.d.). Searching for a face using an image. Amazon. Retrieved from https://docs.aws.amazon.com/ rekognition/latest/dg/search-face-with-imageprocedure.html

Amazon. (2020a). Amazon Rekognition FAQs. Amazon. Retrieved from https://aws.amazon.com/ rekognition/faqs

Amazon. (2020b). Amazon Rekognition customers. Amazon. Retrieved from https://aws.amazon.com/ rekognition/customers

Azar, M. (2018). Algorithmic facial image: Regimes of truth and datafication. A Peer-Reviewed Journal About APRJA, 7(1), 27-35. https://doi.org/10.7146/ aprja.v7i1.115062

Benjamin, R. (2019). Race after technology. Cambridge and Malden, MA: Polity Press

Benjamin, W. (2002). Selected writings. (Vol. 3). Cambridge, MA and London: The Belknap Press of Harvard University.

Benjamin, W. (2003). Selected writings. (Vol. 4). Cambridge, MA and London: The Belknap Press of Harvard University.

Benjamin, W., \& Jennings, M. (2010). The work of art in the age of its technological reproducibility. Grey Room, 39, 11-38.

Blas, Z. (2013). Escaping the face: Biometric facial recognition and the facial weaponization suite. 
Media-N, Journal of the New Media Caucus, 9(2). http:// median.newmediacaucus.org/caa-conferenceedition-2013/escaping-the-face-biometric-facialrecognition-and-the-facial-weaponization-suite

Cheney-Lippold, J. (2017). We are data: Algorithms and the making of our digital selves. New York, NY: New York University Press.

Crawford, K., \& Paglen, T. (n.d.). Excavating Al: The politics of images in machine learning training. Excavating Al. Retrieved from https://www.excavating.ai

Crawford, K., \& Paglen, T. (2019). Training humans. Milano: Fondazione Prada.

Crawford, K., \& Paglen, T. (2020). 'Training Humans' at Osservatorio Pondazione Prada, Milan. Mousse Magazine. Retrieved from http://moussemagazine.it/ kate-crawford-and-trevor-paglen-training-humansat-osservatorio-fondazione-prada-milan-2019-2020

Deleuze, G., \& Guattari, F. (1987). A thousand plateaus: Capitalism and schizophrenia (B. Massumi, Trans.). London and New York, NY: Continuum.

Deng, J., Dong, W., Socher, R., Li, L., Li, K., \& Fei-Fei, L. (2009). ImageNet: A large-scale hierarchical image database. In 2009 IEEE conference on computer vision and pattern recognition (pp. 248-255). New York, NY: IEEE.

Facial recognition is already here: These are the 30+ US companies testing the technology. (2019, June 5). CBInsights. Retrieved from https://www.cbinsights. com/research/facial-recognition-technology-uscorporations

Fazzini, K. (2018, December 6). Amazon's facial recognition service used to scan mugshots, but its also used to track innocuous things like soccer balls. CNBC. Retrieved from https://www.cnbc.com/2018/ 12/06/how-amazon-rekognition-works-and-whatits-used-for.html

Gates, K. (2011). Finding the face of terror. In K. Gates (Ed.), Our biometric future: Facial recognition technology and the culture of surveillance (pp. 97-124). New York, NY: NYU Press.

Hansen, M. (1987). Benjamin, cinema and experience: The blue flower in the land of technology. New German Critique, 40, 179-224.

Howell, C., Strong, J., Ryan-Mosley, T., Cillekens, E., \& Hao, K. (2020, November 18). Citizens are turning face recognition on unidentified police. MIT Technology Review. Retrieved from https://www. technologyreview.com/2020/11/18/1012257/ citizens-are-turning-face-recognition-on-unidentified -police/?fbclid=IwAR3jipN-xicUa-mFUvNokyj49JnsI8 Zbfw33zbBOnidZ5mJQRw0at5ZbVXE

Hristova, S. (2021). In the presence of algorithms. In S. Hristova et al. (Eds.), Algorithmic culture: How algorithms and big data are transforming everyday life.
Lanham, MD: Lexington Books.

Katz, L. (2014, May 8). Anti-surveillance mask lets you pass as someone else. CNET. Retrieved from http://www.cnet.com/news/urme-anti-surveillancemask-lets-you-pass-as-someone-else

McGregory, K., \& Bedy, N. (2020, September 3). Targeted. Tampa Bay Times. Retrieved from https:// projects.tampabay.com/projects/2020/ investigations/police-pasco-sheriff-targeted/ intelligence-led-policing

Michelsen, L. (2018). Thinking beyond biometrics: A playful dance. APRJA, 7(1), 38-52. https://doi.org/ 10.7146/aprja.v7i1.115063

Monahan, T. (2006). Questioning surveillance and security. In T. Monahan (Ed.), Surveillance and society: Technological politics and everyday life (pp. 1-26). London and New York, NY: Routledge.

Monahan, T. (2015). The right to hide? Anti-surveillance camouflage and the aestheticization of resistance. Communication and Critical/Cultural Studies, 12(2), 159-178.

Morrison, E. (2015). Surveillance society needs performance theory and arts practice. International Journal of Performance Arts and Digital Media, 11(2), 125-130.

Noble, S. (2018). Algorithms of oppression: How search engines reinforce racism. New York, NY: New York University Press.

Paglen, T. (2019). Invisible images: Your pictures are looking at you. Architectural Design, 89(1), 22-27. https://doi.org/10.1002/ad.2383

Pasquale, F. (2015). Black box society: The secret algorithms that control money and information. Cambridge: Harvard University Press.

Schep, T. (2020). How normal am I [Video file]. Retrieved from https://www.hownormalami.eu

Sekula, A. (1986). The body and the archive. October, 39, 3-64.

Selvaggio, L. (2015). UMRE Surveillance: Performing privilege in the face of automation. International Journal of Performance Arts and Digital Media, 11(2), 165-184. http://dx.doi.org/10.1080/ 14794713.2015.1086138

Slack, J., \& Hristova, S. (2020). Why we need the concept of algorithmic culture. In S. Hristova et al. (Eds.), Algorithmic culture: How algorithms and big data are transforming everyday life. Lanham, MD: Lexington Books.

Slack, J., \& Wise, G. (2015). Technology and culture. New York, NY: Peter Lang Publishing.

Zuboff, S. (2019). Surveillance capitalism: The fight for a human future at the new frontier of power. New York, NY: Public Affair. 


\section{About the Author}

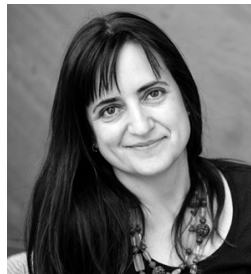

Stefka Hristova is an Associate Professor of Digital Media at Michigan Technological University. She holds a PhD in Visual Studies with emphasis on Critical Theory from the University of California, Irvine. Her research analyzes digital and algorithmic cultures. Hristova is a Co-Editor of Algorithmic Culture: How Big Data and Artificial Intelligence are Transforming Everyday Life (Lexington Books, 2020). Her work has been published in journals such as Visual Anthropology, Radical History Review, tripleC, Surveillance and Security, Interstitial, and Continuum. 